\title{
Effet de la salinité sur la densité des isolats de Pseudomonas spp fluorescents de rhizosphère de plants de tomate, d'aubergine et d'oignon au Sénégal
}

\author{
Demba DIAW*, Mame Arama FALL-NDIAYE, Oubeidillah YOUSSOUFA ALI, \\ Idy Carras SARE et Tahir Abdoulaye DIOP
}

Laboratoire de Biotechnologies des Champignons, Département de Biologie Végétale, Faculté des Sciences \& Techniques, Université Cheikh Anta Diop, BP 5005, Dakar Fann, Sénégal. *Auteur correspondant, E-mail:dembadiaw2001@yahoo.fr ; Tel.: +221775318237

\section{RESUME}

Parmi les microorganismes de la rhizosphère, figurent les Pseudomonas spp fluorescents qui sont des bactéries d'intérêt agricole (Plant Growth Promoting Rhizobacteria). Ces Pseudomonas pourraient permettre le développement de certaines plantes des sols salés et jouer ainsi un rôle important dans la réhabilitation de ces sols au Sénégal. Ainsi l'objectif de cette étude est d'évaluer l'effet de différentes concentrations de $\mathrm{NaCl}$ sur la croissance de Pseudomonas spp fluorescents. Des prélèvements de sol ont été effectués au niveau de la rhizosphère de trois spéculations (oignon, aubergine, tomate) et sur sol nu. Les souches ont été isolées par la méthode des suspensions dilutions, puis ensemencées sur milieu King B. L’identification des isolats a été effectuée par observation sur table UV. Trois isolats ont été sélectionnés et purifiés sur milieu King B pour chaque échantillon de sol. Afin d'évaluer l'effet de la salinité sur ces souches, les concentrations suivantes ont été appliquées : $0 \mathrm{M} ; 0,17 \mathrm{M} ; 0,34 \mathrm{M} ; 0,51 \mathrm{M} ; 0,70 \mathrm{M} ; 0,85 \mathrm{M}$ et 1,03 M. Les résultats obtenus après $72 \mathrm{~h}$ d'incubation montrent que les souches se développent pour des concentrations en $\mathrm{NaCl}$ allant de $0 \mathrm{M}$ à $0,51 \mathrm{M}$. Les concentrations supérieures entraînent une réduction du nombre de colonies pour tous les isolats. Il existe un seuil de tolérance au sel pour chaque souche de Pseudomonas. Cependant, les souches isolées de la rhizosphère de plants d'oignon sont plus résistantes au sel par rapport aux isolats des rhizosphères de plants de tomate et d'aubergine. Ces isolats de Pseudomonas pourraient être utilisés dans les sols salés afin d'améliorer les rendements des cultures.

(C) 2018 International Formulae Group. All rights reserved.

Mots clés: Microorganismes, rhizosphère, PGPR, seuil de tolérance, salinité, résistance.

\section{Effect of salinity on the density of fluorescent Pseudomonas spp isolates from the rhizosphere of tomato, eggplant and onion plants in Senegal}

\begin{abstract}
Among the microorganisms of the rhizosphere are the fluorescent Pseudomonas spp. which are bacteria of agricultural interest (Plant Growth Promoting Rhizobacteria). These Pseudomonas could allow the development of some plants of salty soils and play an important role in the rehabilitation of these soils in Senegal. Then the objective of this study is to evaluate the effect of different concentrations of $\mathrm{NaCl}$ on the growth of fluorescent Pseudomonas spp. So, soil samples were taken from the rhizosphere of three crops
\end{abstract}


(onion, eggplant, tomato) and on bare soil. The strains were isolated by the dilution suspensions method, then seeded on King B medium. The identification of the isolates was performed by observation on a UV table. And three isolates were selected and purified on King B medium for each soil sample. In order to evaluate the effect of salinity on these isolates, the following concentrations were applied: $0 \mathrm{M} ; 0.17 \mathrm{M} ; 0.34 \mathrm{M} ; 0.51 \mathrm{M} ; 0.70 \mathrm{M}$; $0.85 \mathrm{M}$ and $1.03 \mathrm{M}$. The results obtained, after $72 \mathrm{~h}$ of incubation, show that the isolates develop for concentrations between 0 and $0.51 \mathrm{M}$. Higher concentrations lead to significant decrease of colony density for all isolates. There is a salt tolerance limits for each Pseudomonas isolate. However, rhizosphere isolates of onion plants are more resistant to salt than isolates from other rhizospheres. These Pseudomonas isolates could be used in salty soils to improve crop yields.

(c) 2018 International Formulae Group. All rights reserved.

Keywords: Microorganisms, rhizosphere, PGPR, salinity, tolerance-limit, resistant.

\section{INTRODUCTION}

Le sol est un environnement complexe abritant des populations de microorganismes ayant des particularités biologiques et biochimiques diverses. Parmi ces microorganismes, figurent des bactéries d'intérêt agricole (Plant Growth Promoting Croissance) ou PGPR comme les Pseudomonas spp fluorescents. Ces rhizobactéries, Gram négatives de 0,5 à $1 \mu \mathrm{m}$ de diamètre sont mobiles grâce à leurs flagelles polaires (Bell - Perkins et Lynch, 2002). Certaines de ces bactéries sont utilisées en agriculture en tant que biofertilisants et offrent une panoplie d'avantages pour l'obtention de bons rendements agricoles (Ali et al., 2009; Bano et Fatima, 2009; Adjanohoun et al., 2012). En effet, elles peuvent stimuler la croissance des plantes où réduire d'éventuelles maladies causées par les pathogènes comme les champignons, les bactéries, les virus, les nématodes (Rajkumar et al., 2010; Ma et al., 2011) grâce à une compétition pour l'espace et les nutriments (Haas et Defago, 2005). Différents mécanismes, directs (solubilisation du phosphore, du potassium, fixation d'azote, séquestration du fer par les sidérophores, production de régulateurs de croissance...) ou indirects tels que la production d'antibiotiques sont impliqués (Van Loon, 2007 ; Karnwal, 2009).

Cependant, l'activité de ces microorganismes peut être influencée par plusieurs facteurs parmi lesquels la salinisation des sols qui est l'un des facteurs limitant la productivité végétale et le rendement agricole (Baatour et al., 2004 ; Ly et al., 2014). Près d'un milliard d'Ha de terres à travers le monde sont affectés par la salinité, ce qui représente plus de $6 \%$ de la surface du globe (Ould Mohamdi et al., 2011). Ces écosystèmes salés, surtout rencontrés dans les pays arides et semi-arides sont caractérisés par une faible irrégularité des précipitations, associée à une forte évaporation favorisant l'accumulation du sel dans le sol. En Afrique, plus particulièrement au Sénégal, cette salinisation affecte la zone des Niayes qui joue un rôle capital dans l'économie du pays (Ndiaye et al., 2012). Elle représente la principale zone de cultures maraîchères et les principales spéculations concernent le chou, la pomme de terre, la tomate, l'aubergine, la carotte, l'oignon, etc.

L'objectif de notre étude est donc d'évaluer l'effet de différentes concentrations de $\mathrm{NaCl}$ sur la croissance de Pseudomonas spp fluorescents.

\section{MATERIEL ET METHODES}

\section{Isolement des Pseudomonas spp fluorescents}

Des prélèvements de sol ont été effectués au niveau du Centre pour le Développement de l'Horticulture (station de Cambérène, zone éco-géographique des Niayes, Région de Dakar, Sénégal) en période de contre saison (février). Il s'agit de sols provenant de la rhizosphère de plants de tomate, d'oignon et d'aubergine au stade de fructification et sur sol nu. Pour chaque échantillon de sol, $500 \mathrm{~g}$ ont été prélevés sur une profondeur de 0 à $25 \mathrm{~cm}$. A partir de ces 
échantillons, des isolements de Pseudomonas spp fluorescents ont été effectués par la méthode des suspensions-dilutions. Un prélèvement de $1 \mathrm{~g}$ de chaque sol a été mis en suspension dans $10 \mathrm{ml}$ de $\mathrm{MgSO}_{4}, \quad 7 \mathrm{H}_{2} \mathrm{O}$ stérile $0,1 \mathrm{M}$. Après agitation pendant 10 minutes à l'aide d'un vortex, des dilutions décimales $\left(10^{-1}\right.$ à $\left.10^{-8}\right)$ ont été réalisées à partir de cette suspension. $0,1 \mathrm{ml}$ de chaque suspension a été étalé dans des boîtes de Petri contenant chacune $10 \mathrm{ml}$ de milieu gélosé de King B (King et al., 1954). Les boîtes de Petri sont mises à incuber à $28^{\circ} \mathrm{C}$ à l'obscurité.

Au bout de 48 heures d'incubation, les colonies bactériennes ont été observées sous une lampe U.V. à $365 \mathrm{~nm}$. Pour chaque échantillon de sol, trois isolats produisant un pigment fluorescent ont été sélectionnés et purifiés par étalement en stries dans trois boîtes contenant le milieu solide de King B. Chaque isolat est désigné par un code. Il s'agit des souches To, Oi et $\mathrm{Au}$ isolées respectivement de la rhizosphère de plants de tomate, d'oignon et d'aubergine.

\section{Effet du sel sur les Pseudomonas spp fluorescents}

Afin d'évaluer l'effet du sel sur les différents isolats de Pseudomonas spp fluorescents, des milieux KB liquides ont été préparés avec les concentrations salines suivantes : $0 \mathrm{M}, 0,17 \mathrm{M}, 0,34 \mathrm{M}, 0,51 \mathrm{M}, 0,70$ $\mathrm{M}, 0,85 \mathrm{M}$ et $1,03 \mathrm{M}$ à pH 7,2 $\pm 0,2$. Ces milieux ont été autoclavés à $120{ }^{\circ} \mathrm{C}$ pendant 20 minutes. Ensuite, chaque isolat bactérien choisi a été étalé à l'aide d'une micropipette stérile, dans trois des boîtes de Petri contenant chacune $10 \mathrm{ml}$ du milieu KB solidifié. Les boîtes de Petri ont été incubées à $28{ }^{\circ} \mathrm{C}$ à l'obscurité durant $72 \mathrm{~h}$.

\section{Analyse des résultats}

Les résultats ont été analysés avec le logiciel SIGMAPLOT (SIGMASTAT). Ainsi les données ont été traitées statistiquement en adoptant une approche paramétrique qui est l'analyse de la variance (ANOVA). Le test de Newman-Keuls a permis de comparer les moyennes des variables mesurées au seuil de probabilité de 5\%.

\section{RESULTATS}

Isolement

Au bout de $48 \mathrm{~h}$ de culture, les colonies bactériennes ont été observées sur une table U.V. à $365 \mathrm{~nm}$. Les isolats de Pseudomonas spp fluorescents issus des rhizosphères de plants de tomate, d'oignon et d'aubergine ont montré une fluorescence par rapport au sol nu où aucune fluorescence n'a été observée (Figure 1).

\section{Effet du sel sur la densité des isolats de Pseudomonas spp fluorescents}

Les Pseudomonas spp fluorescents provenant de la rhizosphère de tomate, d'oignon et d'aubergine (To, $\mathrm{Oi}, \mathrm{Au}$ ), cultivés sur milieu KB pendant 72 heures ont montré une variabilité du nombre de colonies en fonction de la concentration en $\mathrm{NaCl}$ (Figure 2).

Après $72 \mathrm{~h}$ d'incubation, tous les isolats sont en croissance en l'absence de $\mathrm{NaCl}(0 \mathrm{M})$. Il $\mathrm{n}$ y a pas de différence significative entre le nombre de colonies provenant de la rhizosphère d'aubergine $(\mathrm{Au})$ et celui de la rhizosphère de tomate (To). Les valeurs obtenues sont deux fois supérieures à celles du nombre d'isolats provenant de la rhizosphère d'oignon $(\mathrm{Oi})$.

Cette croissance se poursuit pour des concentrations en $\mathrm{NaCl}$ allant de $0,17 \mathrm{M}$ à 0,51 M. Cependant, une baisse a été notée pour les colonies provenant de la rhizosphère d'oignon à $0,17 \mathrm{M}$ et celles de la rhizosphère de tomate à $0,51 \mathrm{M}$. Par contre, à $0,34 \mathrm{M}$, la croissance est optimale pour tous les isolats et il $\mathrm{n}$ y a pas de différence significative pour l'ensemble des isolats avec un nombre moyen de colonies égal à 303.

Pour des concentrations en $\mathrm{NaCl}$ allant de $0,7 \mathrm{M}$ à $0,85 \mathrm{M}$, on note une baisse significative du nombre de colonies pour la rhizosphère de plants de tomate.

Lorsque la concentration en $\mathrm{NaCl}$ est de $1,03 \mathrm{M}$, il y a une inhibition totale et les isolats ne se développent pas à l'exception de ceux provenant de la rhizosphère de plants d'oignon avec un nombre de colonies très faible. 


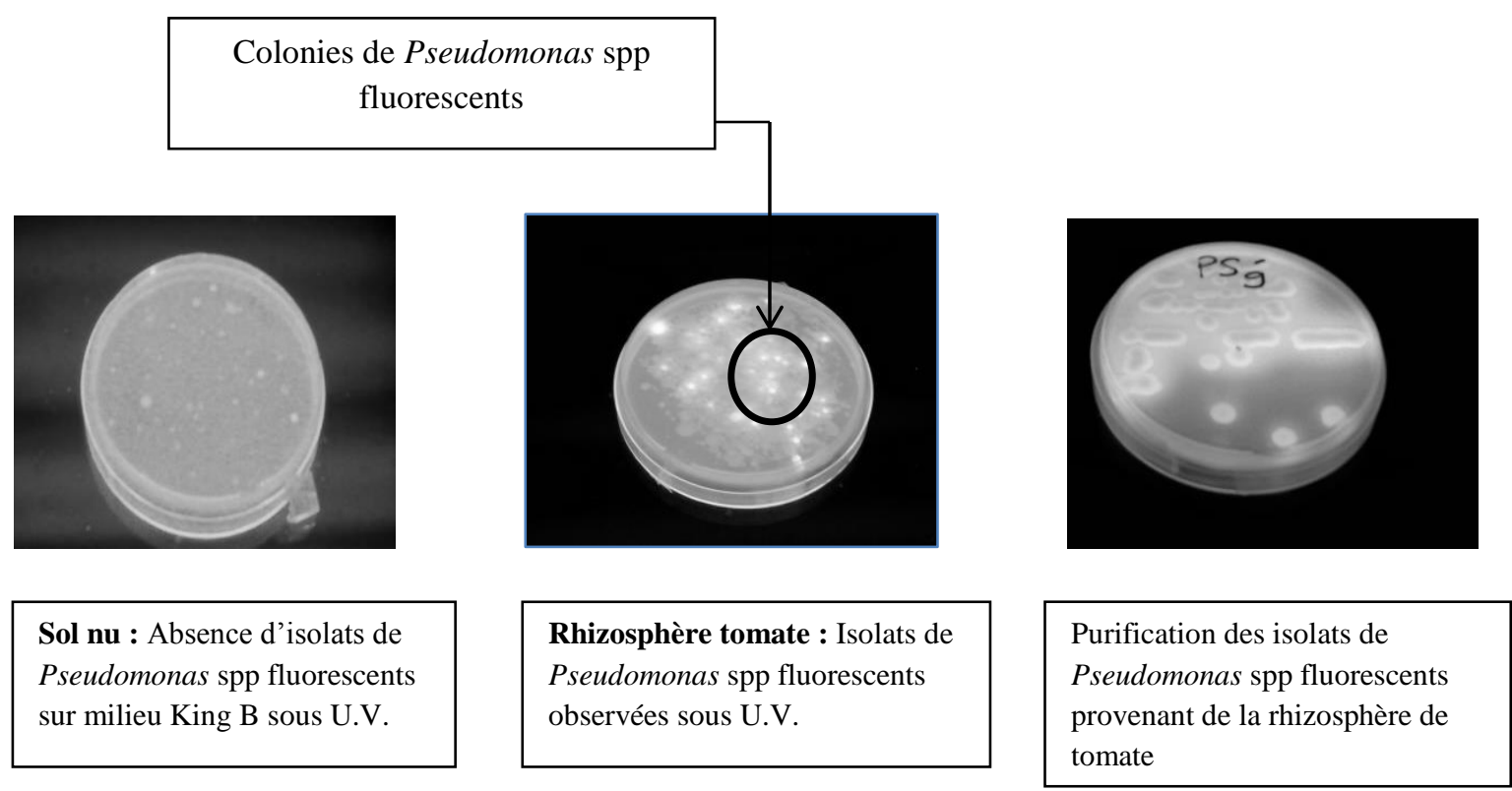

Figure 1 : Isolats de Pseudomonas spp fluorescents isolées de la rhizosphère de tomate.

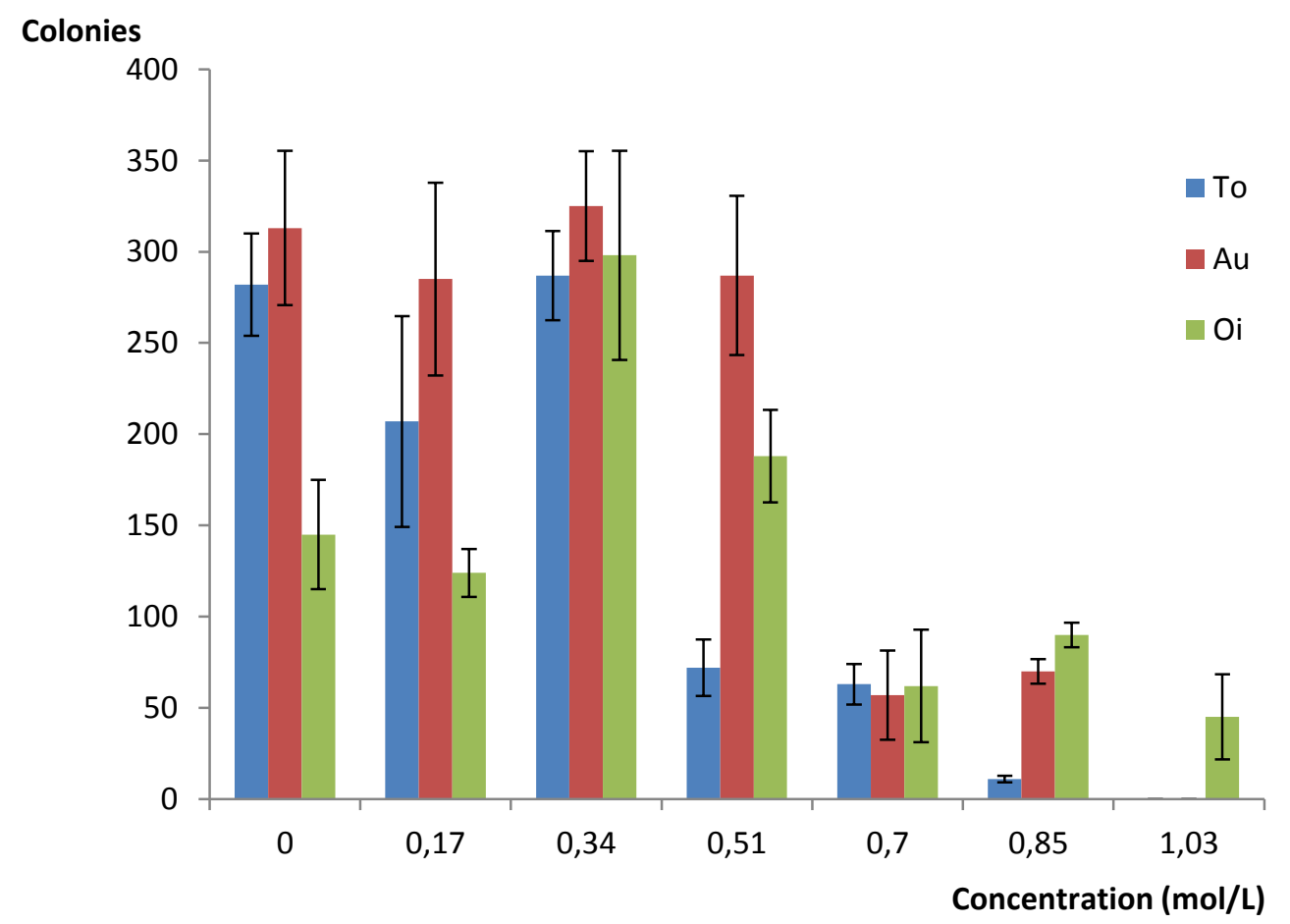

Figure 2: Effet de différentes concentrations de $\mathrm{NaCl}$ sur le nombre de colonies de Pseudomonas spp fluorescents. 


\section{DISCUSSION}

Les résultats obtenus dans des conditions in vitro ont montré que les rhizosphères de plants de tomate, d'aubergine et d'oignon renferment des Pseudomonas spp fluorescents contrairement au sol nu qui n'en contient pas. Ces résultats confirment l'effet rhizosphère qui a été décrit par plusieurs auteurs (Samia, 2012). Cet environnement est caractérisé par des interactions entre les exsudats racinaires et les microorganismes (Bell-Prekins et Lynch, 2002). Ces exsudats racinaires incluent la sécrétion d'ions, d'oxygène libre, d'eau, d'enzymes, de mucilages ainsi qu'un grand nombre de métabolites primaires et secondaires (Bertin et al., 2003). Ces rhizobactéries trouvent ainsi dans la rhizosphère de ces plantes, un grand nombre de nutriments favorables à leur développement (Rajkumar et al., 2010); ce qui expliquerait leur absence dans les sols nus.

Cependant, le développement des Pseudomonas spp fluorescents peut être influencé par la salinité. Les souches de Pseudomonas spp isolées de la rhizosphère de tomate, d'aubergine et d'oignon ont montré une variabilité du nombre de colonies en fonction des différentes concentrations en $\mathrm{NaCl}$. Toutes les souches tolèrent le sel jusqu'à $0,34 \mathrm{M}$. Ces résultats sont en accord avec ceux de Deshwal et Kumar (2013) qui ont montré que la plupart des microorganismes ont des besoins limités en sels et sont capables même de croître pour des concentrations en $\mathrm{NaCl}$ inférieures à $0,3 \mathrm{M}$. Les teneurs en $\mathrm{NaCl}$ de $0,51 \mathrm{M}$ à 0,85 ralentissent fortement la croissance des souches de Pseudomonas spp fluorescents. Ce phénomène est dû au fait que les teneurs en $\mathrm{NaCl}$ supérieures à $0,51 \mathrm{M}$ entraînent un choc hyper-osmotique pour les cellules vivantes du fait de la perméabilité des membranes cellulaires provoquant une plasmolyse suite à une augmentation de la teneur en sel. Cette plasmolyse aboutit à l'inhibition de certaines fonctions cellulaires comme l'absorption des nutriments, la réplication de l'ADN ou la biosynthèse de macromolécules, ce qui provoque la mort des cellules (Saum et Müller, 2007). Ces mêmes résultats ont été prouvés par Houda (2011) qui a montré que les concentrations en $\mathrm{NaCl}$ de 0,5 M entrânent une diminution significative de la croissance de certaines souches de Pseudomonas fluorescents, voire même une inhibition. Par contre, ces résultats sont contradictoires avec ceux de Deshwal et Kumar (2013) qui ont montré que certaines souches de Pseudomonas ont une tolérance au sel pour des teneurs ne dépassant pas 0,2 M.

\section{Conclusion}

Les rhizosphères de plants de tomate, d'oignon et d'aubergine abritent plusieurs espèces de Pseudomonas spp fluorescents. Cependant, le comportement des Pseudomonas spp fluorescents peut être directement influencé par la salinité. Les isolats de Pseudomonas spp fluorescents provenant de la rhizosphère des plants d'oignon sont plus résistants au sel par rapport à ceux des rhizosphères de plants d'aubergine et de tomate. Il existe une limite de tolérance vis à vis $\mathrm{du} \mathrm{NaCl}(0,51 \mathrm{M})$ et au-delà de cette concentration, on a une baisse significative du nombre de colonies. La tolérance vis-à-vis du sel est donc un caractère propre à chaque souche de Pseudomonas. Ces isolats de Pseudomonas pourraient être utilisés dans les sols salés afin d'améliorer les rendements des cultures dans ces zones.

\section{CONFLITS D'INTERETS}

Les auteurs déclarent n'avoir aucun conflit d'intérêts.

\section{CONTRIBUTIONS DES AUTEURS}

DD est l'auteur principal ayant réalisé la majeure partie de ce travail de recherche. Il est intervenu dans l'élaboration et la mise au point du protocole expérimental, l'analyse et l'interprétation des données et à la rédaction entière de l'article. OYA est intervenu dans l'élaboration et la mise au point du protocole expérimental, l'analyse et l'interprétation des données. MAF-N et ICS ont apporté leur contribution à la révision du contenu sur le fond que sur la forme. TAD est le superviseur de ce travail de recherche. Il est intervenu dans la correction du protocole, et a coordonné toutes les activités.

\section{REFERENCES}

Adjanohoun A, Noumavo PA, SIKIROU R, Allagbe M, Gotoechan-Hodonou H, Dossa KK, Yehouenou B, Glele Kakaï R, BabaMoussa L. 2012. Effets des rhizobactéries PGPR sur le rendement et les teneurs en macroéléments du maïs sur sol ferralitique non dégradé au Sud Bénin. Int. J. Biol. Chem. Sci., 6(1): 279-288. DOI: http://dx.doi.org/10.4314/ijbcs.v6i1.24 
Ali SZ, Sandhya V, Grover M, Kishore N, Rao LV, Venkateswarlu B. 2009. Pseudomonas sp. strain AKM-P6 enhances tolerance of sorghum seedlings to elevated temperatures. Biol. Fert. Soils, 46: 45-55. DOI: http://dx.doi.org/10.1007/s00374-0090404-9

Baatour O, M'rah S, Ben Brahim N, Boulesnem F, Lachaal M. 2004. Réponse physiologique de la gesse (Lathyrus sativus) à la salinité du milieu. Revue des Régions Arides, 1 ( ${ }^{\circ}$ Spécial) : 346-358.

Bano A, Fatima M. 2009. Salt tolerance in Zea mays (L.) following inoculation with Rhizobium and Pseudomonas. Biol. Fert. Soils, 45: 405-413. DOI: http://dx.doi.org/10.1007/s00374-0080344-9

Bell-Perkins LJ, Lynch JM. 2002. Rhizosphere microbiology, p. 2713-2728. In Encyclopedia of environmental microbiology, Bitton G (ed.). A WileyInterscience Publication: Canada. DOI: http://dx.doi.org/10.1002/0471263397.env 161

Bertin C, Yang XH, Weston LA. 2003. The role of root exudates and allelochemicals in the rhizosphere. Plant and Soil, 256: 6783. http://dx.doi.org/10.1023/A:1026290508166

Deshwal VK, Kumar P. 2013. Effect of salinity on growth and PGPR activity of Pseudomonads. Journal of Academia Industrial Research, 2: 353 - 356.

Haas D, Defago G. 2005. Biological control of soil-borne pathogens by fluorescent pseudomonads. Nat. Rev. Microbiol., 3(4): 307-319. http://dx.doi.org/10.1038/nrmicro1129

Houda RE. 2011. Isolement de Pseudomonas spp fluorescents d'un sol sale. Effet d'osmoprotecteurs naturels. Magistère, Université Ferhat Abbas Setif (Algérie). $121 \mathrm{p}$.

Karnwal A. 2009. Production of indole acetic acid by fluorescent Pseudomonas in the presence of L-Tryptophan and rice root exudates. J. Plant Pathol., 91: 61-63. DOI: http://dx.doi.org/10.4454/jpp.v91i1.624

King EO, Ward MK, Raney DE. 1954. Two simple media for the demonstration of pyocyanin and fluorescein. J. Lab. Clin. Med., 44: 301-307.
Ly MO, Kumar D, Diouf M, Nautiyal S, Diop T. 2014. Effet de la salinité sur la croissance et la production de biomasse de deux provenances de Jatropha curcas L. cultivés en serre. Int. J. Biol. Chem. Sci., 8(1): $\quad 46-56 . \quad$ DOI : http://dx.doi.org/10.4314/ijbcs.v8i1.5

Ma Y, Prasad MNV, Rajkumar M, Freitas H. 2011. Plant growth promoting rhizobacteria and endophytes accelerate phytoremediation of metalliferous soils. Biotechnology Advances, 29: 248-258. DOI:

http://dx.doi.org/10.1016/j.biotechadv.201 0.12 .001

Ndiaye O, Diallo A, Matty F, Thiaw A, Fall RD, Guissé A. 2012. Caractérisation des sols de la zone des Niayes de Pikine et de Saint Louis (Sénégal). International Journal of Biological and Chemical Sciences, 6: 519-528. DOI: http://dx.doi.org/10.4314/ijbcs.v6i1.46

Ould Mohamdi M, Bouya D, Ould Mohamed Salem A. 2011. Etude de l'effet du stress salin $(\mathrm{NaCl})$ chez deux variétés de tomate (Campbell 33 et Mongal). Int. J. Biol. Chem. Sci., 5(3): 860-900. DOI: http://dx.doi.org/10.4314/ijbcs.v6i4.30

Rajkumar M, Ae N, Prasad MNV, Freitas H. 2010. Potential of siderophore-producing bacteria for improving heavy metal phytoextraction. Trends in Biotechnology, 28: 142-149. DOI: http://dx.doi.org/10.1016/j.tibtech.2009.12.002

Samia MM. 2012. Localisation des déterminants de la suppression de quelques souches de Pseudomonas isolées de la rhizosphère de la pomme de terre. Thèse de doctorat en sciences, Université Ferhat Abbas de Sétif 1, Algérie.

Saum SH, Müller V. 2007. Salinity-dependant switching of osmolyte strategies in a moderately halophilic bacterium: Glutamate induces proline biosynthesis in Halobacillus halophilus. J. Bacteriol., 189: 6968-6975. DOI: http://dx.doi.org/10.1128/JB.00775-07

Van Loon LC. 2007. Plant responses to plant growth-promoting rhizobacteria. Eur. J. Plant Pathol., 119: 243-254. DOI: http://dx.doi.org/10.1007/s10658-0079165-1. 